\title{
The impact of the Hurst parameter on queueing performances using chaotic sequences
}

\author{
Authors: E. Costamagna, L. Favalli, P. Gamba, G. Iacovoni ${ }^{(*)}$ \\ Dipartimento di Elettronica, Università di Pavia, Italy \\ (*) Also with CORITEL, Rome, Italy \\ e-mail: giovanni@comell.unipv.it \\ tel: 06-204110032-fax: 06-20410037
}

\begin{abstract}
The need to understand the nature of packet traffic is growing more and more as its volume is increasing, due to the development and the deployment of optical network technologies. The Hurst parameter is the key figure which captures the degree of Long Range Dependence in the new traffic paradigms. Its impact on network engineering is still unclear and object of a strong debate, especially after the discovery of the so-called "cross-over effect".

In order to give a contribution to this discussion, in this paper we compare the queueing simulation results when an infinite buffer is loaded with different sequences, each sequence having a different Hurst parameter value, but the same marginal distributions. Simulations have been carried out using different phase-shifts and load conditions.
\end{abstract}

\section{Keywords}

ATM, Hurst parameter, Long range dependence, Self-similarity

\section{INTRODUCTION}

The volume of traffic carried over telecommunication networks is increasing, following the development and the deployment of optical technologies. A significant part of this traffic is transported and switched using a packet-like structure. The need to understand the nature of packet traffic is growing more and more as it gives birth to difficult engineering problems 


\section{Part Six: Traffic Modeling}

such as bandwidth allocation and buffer sizing.

Through extensive measurements on working packet networks, the fractal-like characteristics (or self-similar properties) of aggregated packet streams, whatever the type of traffic (Ethernet's LANs, VBR video, ISDN control channel, etc.), has recently been shown. A key feature of self-similar processes is the "Hurst effect" or "persistence", i.e. the correlation between records observed over different time scales; this effect can be captured by the so called Hurst-parameter $\mathrm{H}$.

More precisely, let $\left\{X_{i}\right\}$ be a covariance stationary process and let $\left\{X(m)_{i}\right\}$ be the aggregated process obtained by averaging the original series over non-overlapping intervals of size $m$; then there exists $H$ (the Hurst parameter) such that:

$$
\operatorname{var}\{X(m)\} \sim m^{-2(1-\mathrm{H})} \quad \text { as } m \rightarrow \infty \quad(1 / 2 \leq \mathrm{H}<1)
$$

If $\mathrm{H}=0.5$ the process has a short-range dependence ( $\mathrm{SRD}$ ), whereas $\mathrm{H}>0.5$ characterizes a process with long range dependence (LRD), also called asymptotically self-similar. If $\operatorname{var}\{X(m)\}=m^{-2(1-H)}$ is true for all $m \geq 1$, then the process is exactly self-similar, as for instance the Fractional Brownian Motion (FBM).

The Hurst parameter can therefore be put in relation with the behaviour of the process when it is considered its low frequencies spectra.

After the above mentioned discovery of fractal-like characteristics (which implicate LRD) in teletraffic, there has been a strong debate whether or not engineering formulae can still be based on traditional markovian models, known to be SRD. Someone claims [1] that the latters lead to underestimation of the bandwidth requirements, while other researches [2] show a succesful use of markovian models.

The discrepancy between the two above sets of seemingly contradictory results has been explained through the help of the "cross-over" effect: when a large enough number of i.i.d. sources are multiplexed, larger multiplexing gains are obtained with high-H sources than with low-H sources. More precisely, for FBM traffic, in[3] it has been shown that, given two FBM streams with the same mean $\mu$ and the same variance $\sigma$ but with different Hurst parameters $\mathrm{H}_{1}$ and $\mathrm{H}_{2}$ then for a finite level $\mathrm{B}$ in an infinite buffer, there exists a value $\mu^{*}$ such that for $\mu \geq \mu^{*}$ the stream with the higher $H$ requires smaller bandwidth to meet a specified overflow probability requirement.

Through simulations, the same cross-over effect was observed in [4] where the performances of VBR video traces (which are only asymptotically self-similar) were compared with those of a finite-state markovian model: a large number of multiplexed i.i.d. sources requires less bandwidth when each source has $\mathrm{H}=0.7$ than when it has $\mathrm{H}=0.5$.

In order to better understand the engineering significance of the $\mathrm{H}$ parameter, in section II of this paper we present the queueing simulation results for an infinite buffer loaded each time with a different sequence. These sequences are derived from a set of three traces; each trace 
has a different $\mathbf{H}$, but all of them exhibit the same marginal distribution, i.e. the number of packets in a GOP period (one GOP = 12 video frames, each one lasting 1/25 sec). One trace is a real VBR video, while the other two have been built using chaotic maps; in a previous work [5] we validated the modelling capability of the two chaotic traces by comparing the QoS parameters of an ATM multiplexer of finite size fed by the modelled traces with those resulting from the actual VBR video load.

In section III we briefly discuss the results of these simulations, emphasising the role played by the $\mathrm{H}$ parameter.

\section{SIMULATION RESULTS}

The VBR video trace is the cartoon ASTERIX, MPEG coded, with high activity [for a discussion of activity see 6], since $\mathrm{H}=0.93$. The two chaotic traces (CAOS_1 and CAOS_2, for detail refer to [5]), built by sampling the trajectory of the Lorenz attractor, simulate ASTERIX at the Group of Picture Level (GOP). This modelling approach is similar to that described in [7], where chaotic mechanisms are used to simulate the physical process of channel error generation.

Some relevant statistical properties are summarized in the following table:

Table 1 Statistical properties of viedo trace

\begin{tabular}{llll}
\hline & Mean $(\mathrm{kbit} / \mathrm{sec})$ & $\sigma(\mathrm{Kbit} / \mathrm{sec})$ & $\mathrm{H}$ \\
\hline MPEG & 558 & 259 & 0.81 \\
CAOS_1 & 558 & 259 & 0.68 \\
CAOS_2 & 558 & 259 & 0.78 \\
\hline
\end{tabular}

H values have been calculated using time-variance plots ([6]); this is just a statistical method which tries to estimate an asymptotic behavior from a finite data set. Therefore these values must be considered with care.

Each trace is packetized in ATM cells introducing AAL5 overhead (one AAL5 PDU made of 8 ATM cells). The cells are then evenly spaced over a GOP period; this, however, is not critical since it has been noted that only low frequencies in the power spectra dominate queueing behaviour [8].

In [5] we showed that when feeding finite buffers with deterministic service time, FIFO mode, with CAOS_2, the obtained QoS parameters were highly consistent with those obtained with the MPEG trace for buffer size up to 100 cells; we related this result to the good 
matching on the time variance plot of the two sequences for low lags (fig. 1). In the same way, CAOS_1, which matches the MPEG values for larger values of lags (fig. 2) gave good results for 10,000 cells buffer.

Now we extend the simulation of queueing performances to the three sequences feeding an infinite buffer. First, for the sequence $\left\{X_{\mathrm{i}}\right\}$ corresponding to the MPEG trace we produce the new sequence:

$$
Y i=\sum_{j=1}^{j=6} X(i+n j)
$$

For $\mathrm{n}=10$ this leads to the sequence MPEG_SHIFT10, while $\mathrm{n}=100$ leads to the sequence MPEG_SHIFT100; in other words we obtain two phase-shifted versions of the same MPEG stream. We give the following two figures in order to have an approximate idea about the degree of MPEG autocorrelation corresponding to these two phase-shifts: at 10 lags it is still remarkable (0.36), while at 100 lags it has reached quite a small value $(0.1)$.

Similarly we produced on one hand CAOS_1_SHIFT10 and CAOS_1_SHIFT100, and on the other hand CAOS_2_SHIFT10 and CAOS_2_SHIFT100.

Each of the 9 sequences is sent to the infinite ATM buffer; the outgoing data frequency is varied so as to consider a load ranging from $10 \%$ to $100 \%$ (with $10 \%$ step size): this should correspond to varying the number of multiplexed streams when the service time is kept constant.

For phase-shift $=0$, fig. $3 \mathrm{a}$ depicts the obtained mean queue length (mql) versus load for the three sequences; similarly fig. $3 \mathrm{~b}$ and $3 \mathrm{c}$ show the mql for SHIFT10 and SHIFT100 respectively. Besides, for three values of load $(50 \%, 70 \%, 100 \%)$, fig 4 and 5 show the complementary queue length distributions for the chaotic sequences compared to those of the actual MPEG traces, when no shift is applied. Similar results (not shown here for brevity) are obtained when considering the delay distributions.

\section{DISCUSSION}

Here are some observations derived from the results:

1) For loads up to $40 \%$ and greater than $80 \%$ the chaotic sequences exhibit a behaviour similar to the original traces. Besides, the chaotic sequence CAOS_1_NO_SHIFT mimic quite well the MPEG_NO_SHIFT sequence in fig. 4; similarly, the mean queue length of CAOS_1 and that of the MPEG sequences are almost always the same (fig. 3). This supports the feeling that the behavior of the chaotic sequences is well matched to the simulated traffic for a proper choice of the attractor parameters, load and phase-shift 
2) Cross-over effects can actually be observed (see for instance fig. 4). However more crossing points between two curves (see for instance fig. 4a) can be noticed and, strikingly, cross-overs can take place several times as the load is increased. A better definition of crossover effect is therefore needed

3) Apparently the phase-shift results in separating the chaotic curves from the original one, but only for average loads; the larger the phase-shift (and therefore the less the degree of correlation), the larger the distance. Anyway, the phase-shift has a considerable impact on cross-over effects, and it deserves further studies

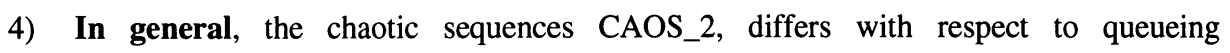
performance from the MPEG sequences, even if the $\mathrm{H}$ parameters are very similar $(0.81$ and 0.78): see fig. 3 and 5. On the other hand the CAOS_1 $(\mathrm{H}=0.68)$ sequence exhibits a closer behavior to that of the MPEG one (see for instance the result for the mean queue length, fig. 3). A reason for this could be found in the similarity of the actual values in time-variance plot for large lags, and not in the similarity of the $\mathrm{H}$ parameters.

Obviously, all these questions and these preliminary results need further investigation; therefore we intend to perform new simulations, with aggregated traffic and other scenarios (type of traces, other $\mathrm{H}$ values, etc.).

Thanks to Marco Isopi (University of Rome La Sapienza) for useful discussions.

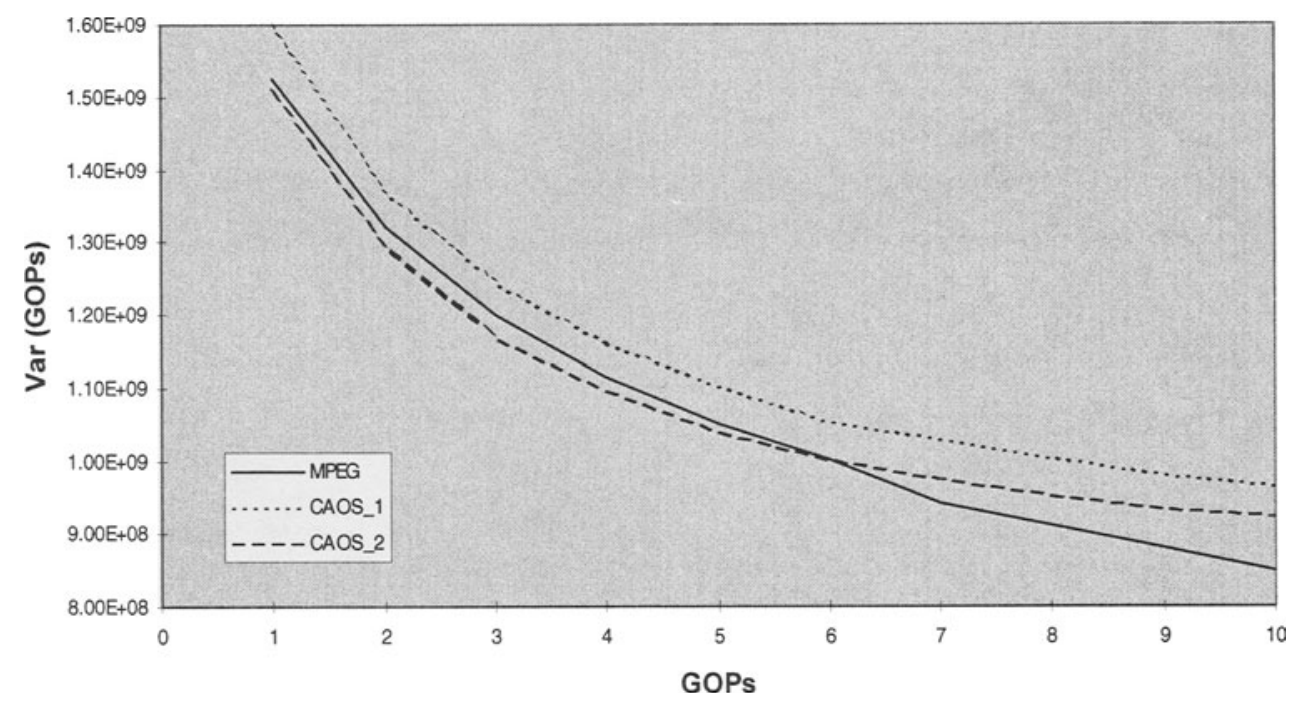

FIG.1. Variance-time plot (linear scale) in the range [0:10] 
136 Part Six: Traffic Modeling

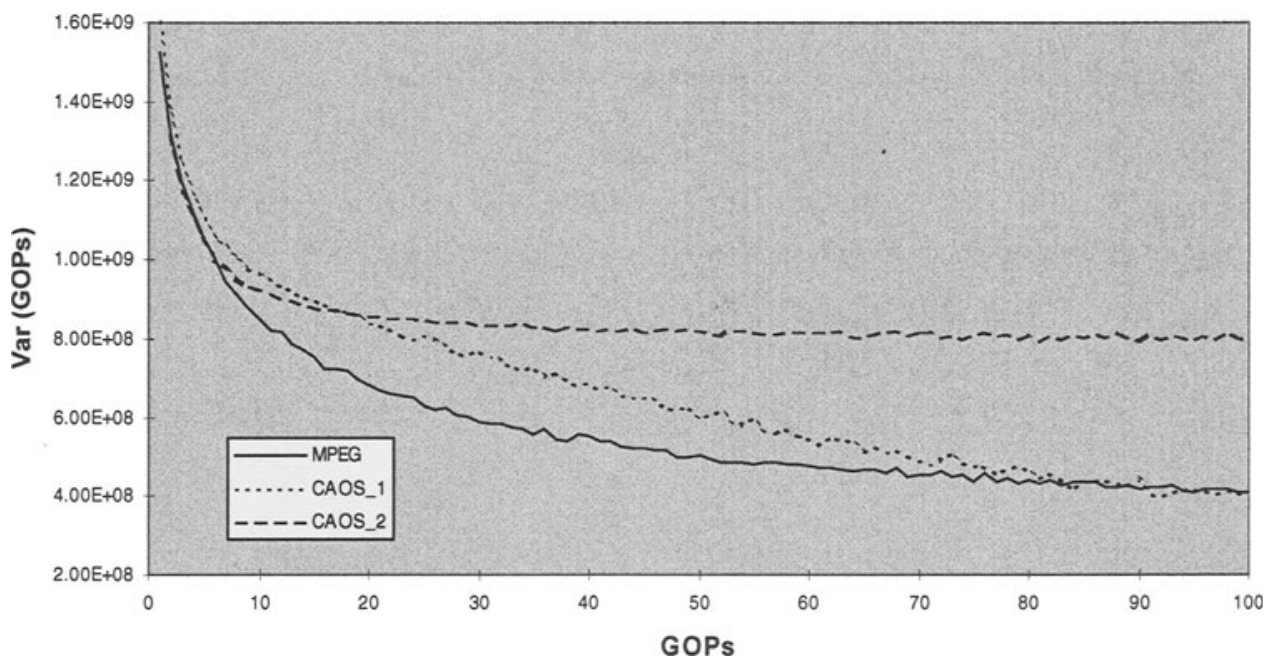

FIG.2. Variance-time plot (linear scale) in the range [0:100]

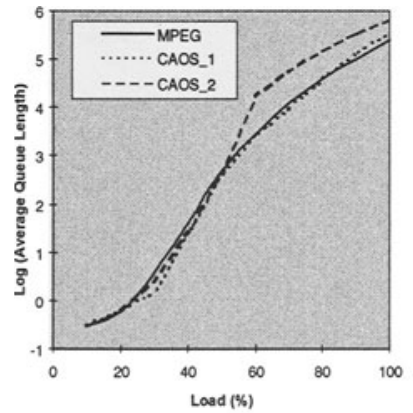

a) phase shift $=0$



b) phase shift $=10$

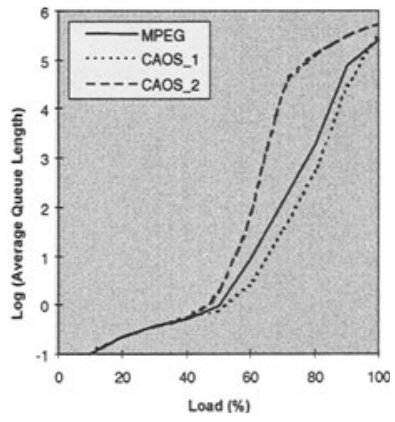

c) phase shift $=100$

FIG.3. Average queue length 


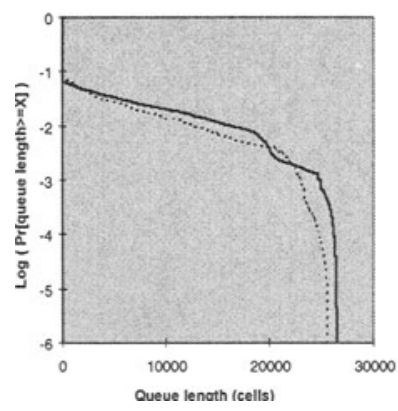

a) $\mathrm{load}=50 \%$



b) $\mathrm{load}=70 \%$

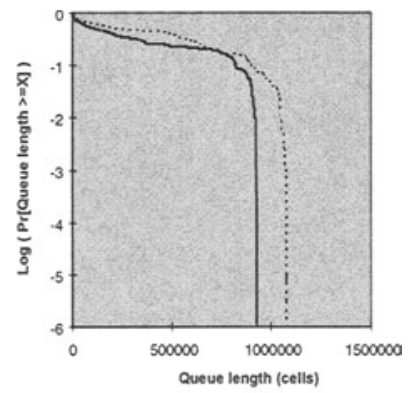

c) $\mathrm{load}=100 \%$

FIG.4. Complementary distribution function (MPEG: continuos line; CAOS_1: dotted line)



a) $\mathrm{load}=50 \%$



b) load $=70 \%$



c) $\operatorname{load}=100 \%$

FIG.5. Complementary distribution function (MPEG: continuos line; CAOS_2: dashed line)

\section{References}

[1] A. Erramilli, O. Narayan, W. Willinger: "Experimental queueing analysis with long-range dependent packet traffic”, IEEE Tr. On Networking, 4, 2, 1996

[2] D.P. Heyman, T.V. Lakshman, "What are the implications of long-range dependence for VBRvideo traffic engineering?", IEEE Tr. On Networking, 4, 3, 1996

[3] K.R. Krishnan, "A new class of performance result for fractional brownian traffic model", Queueing System 22, 1996

[4] K.R. Krishnan, G. Meempat, "Long-range dependence in VBR video streams and ATM traffic 
138 Part Six: Traffic Modeling

enginering", Performance Evaluation, 30, 1997

[5] E. Costamagna, L. Favalli, P. Gamba, G. Iacovoni: "A simple model for VBR traffic based on chaotic maps: validation through evaluation of ATM multiplexers QoS parameters", IEEE-ICC '98, accepted for publication

[6] J. Beran, R. Sherman, M.S. Taqqu, W. Willinger: "Long-range dependence in variable bit rate video traffic", IEEE Tr. On Communications, 43, 2/3/4, 1995

[7] G. Benelli, E. Costamagna, L. Favalli, P. Gamba: " Satellite channels modelled by chaotic bit error generators", EMPS '96 , Rome, Italy, Oct. 1996

[8] S. Li, J. D. Pruneski, "The linearity of low frequency traffic flow: an intrinsic property in queueing systems", IEEE Tr. On Networking, 5, 3, 1997 